\title{
ASSESSING THE EFFECT OF ONLINE HOMEWORK ON STUDENT LEARNING IN A FIRST CIRCUITS COURSE
}

\section{Dr. Katie Evans, Louisiana Tech University}

Dr. Katie Evans is the Walter Koss Endowed Associate Professor of Mathematics and Statistics and the Interim Director of Mathematics and Statistics and Industrial Engineering programs. She is the Director of the Integrated STEM Education Research Center (ISERC) and the Director of Louisiana Tech's Grand Challenge Scholars Program. She earned her Ph.D. in Mathematics and M.S. in Mathematics at Virginia Tech, Blacksburg, VA. Her research interests include distributed parameter control modeling and simulation, dynamic modeling of physical systems, and STEM education. She has published 20 peer-reviewed publications in these areas, and her research has been funded by the NSF, AFRL, and LA-BOR. She also serves as an Associate Editor for the American Control Conference and the Conference on Decision and Control, two premier conferences in the controls community. She is a member of the IEEE, SIAM, and ASEE.

\section{Dr. Paul Hummel, Louisiana Tech University}

Paul Hummel is a lecturer in the Electrical Engineering department at Louisiana Tech University. He has a BS in Engineering with a Computer concentration from LeTourneau University and a PhD in Engineering with an emphasis on Microelectronics from Louisiana Tech University. His current activities focus on project based learning and online student assessment. 


\section{ASSESSING THE EFFECT OF ONLINE HOMEWORK ON STUDENT LEARNING IN A FIRST CIRCUITS COURSE}

\section{Introduction}

To meet the needs of today's students and to maximize efficient use of faculty resources, electronically delivered homework is becoming ever more popular in higher education. In mathematics, for example, online homework can be found for a wide range of courses at a variety of schools, ranging from community colleges to Ivy League universities. Some institutions rely on commercially available packages for homework delivery, while others write their own homework software in-house or employ freely available systems. The authors' institution has considerable experience with the open-source, freely available homework delivery tool WeBWorK. WeBWorK's use in mathematics has been well-established, with it now being employed at over 800 institutions worldwide.

As part of a currently funded National Science Foundation (NSF)-funded project, our team is expanding the use of WeBWorK to engineering courses. Specifically, we are targeting the development of homework problems for three core semester-long, sophomore-level engineering courses: Statics and Mechanics of Materials, Electrical Engineering and Circuits I, and Thermodynamics. Following sufficient debugging and testing, these problems will be housed in a National Problem Library maintained by WeBWorK, with the support of the Mathematical Association of America (MAA) and the NSF.

Though the trend of online homework in higher education continues to emerge, it is important to note that the effects of online homework in engineering have only been explored to a limited degree by the engineering education community. Therefore, our team is also studying the effect of online engineering homework on student learning. The focus of this paper is a study conducted when implementing online homework in a first circuits course during the Winter 2013-14 term. This paper provides background information on WeBWorK, as an open-source homework system, discusses the pedagogical strategies of the homework development, and examines the effect of the online engineering homework on student learning.

\section{WeBWorK Background}

Although an electronic homework system is not a new concept, its application in engineering education is a relatively new area with considerable work remaining to be done. In recent years, there has been an increase in the level of competition among publishing companies and the commercial sector to develop online homework and tutorial systems for lower level engineering courses. Tools like individualized homework, automatic assignment grading, instantaneous student feedback, videos, tutorials, etc., which have long existed in mathematics, for example, are now becoming available for core courses in engineering for a fee.

Commercial packages advertise that instructors may write their own problems for inclusion in homework sets or a problem library, but it has been our experience that this is not necessarily an intuitive process. Further, if instructors write their own problems, there appears to be no fee discount offered to students, even though the company did not actually pay for the development 
of those problems. In other words, an instructor's contribution does not remain open source. Another hindrance of any commercially available homework system is that an instructor is then indefinitely tied to a particular textbook and/or publishing company.

One answer to the weaknesses identified here is an open source homework solution, in which problems are written by faculty and made available free of charge in the public domain.

WeBWorK is such homework system and one in which the project team has considerable experience. WeBWorK was developed by Professors Michael Gage and Arnold Pizer of the University of Rochester. They developed the tool in 1996 as a mechanism for effectively and efficiently delivering online homework to mathematics courses. Now, over 800 institutions, ranging from high schools to $\mathrm{PhD}$-granting universities, have successfully implemented WeBWorK in at least some of their mathematics courses ${ }^{1}$. In recent years, WeBWorK has been implemented in 40 countries outside the United States. A few institutions have also started using WeBWorK in their physics and computer science courses.

WeBWorK introductory circuits problems have been created and used at Northern Arizona University, though these problems have not yet been made available to the public ${ }^{2}$. Additionally, Dr. Joel Trussell, Editor-in-Chief of the Proceedings of the IEEE and Professor of Electrical and Computer Engineering (ECE) at North Carolina State University, has implemented WeBWorK in a sophomore circuits course and is working to expand its usage to other courses. Professor Trussell's recent article also summarizes the state of computer-based homework in the ECE community ${ }^{3}$.

\section{Faculty Impact}

From the administrative side, WeBWorK uses resources efficiently. WeBWorK implementation requires modest physical resources, and it is relatively simple to keep up to date over time. Once problem sets are implemented, it is easy to use them 1) between different sections of a course, 2) with different teachers, 3) for an indefinite period of time, 4) even with changes in textbooks. That is, once problem sets are created, it is not necessary to update the sets from one term or year to the next or when a publishing company releases a new edition of a textbook. Instructors also have the flexibility to change textbooks entirely without the need to entirely recreate their course's homework sets. In this situation, usually only a reordering or regrouping of problems is necessary so that assignments would correspond to sections in the newly chosen text. All of the software required to run WeBWorK is available at no cost because it is open-source. The program does require an appropriate web server, but no special computing equipment is needed by any of the faculty or students who are involved with WeBWorK. All of the additional day-today activities involved can be done through any web browser. Moreover, WeBWorK reduces the amount of paper grading by instructors. When the solution process or format of the submission accounts for part of the homework grade, there may still be a need for modest paper grading by the instructor. An instructor may even choose to assign open-ended projects to assess conceptual skills and understanding of processes that cannot be ascertained through online homework assignments. Overall, the implementation of WeBWorK gives faculty the opportunity to redirect their time towards other efforts that improve student learning as well as other university responsibilities. One faculty comment, in particular, summarizes our observations, 
"As we no longer have funds for graders, I would give much less (perhaps even no) homework if I did not have Webwork. With Webwork, and in particular with its instant feedback, I am able to assign homework for every class period."

\section{Homework Problem Development}

WeBWorK problems are written in a parameterized fashion. A pseudo-random number generator is used to create different problem sets for each student. Students may work together to solve their homework because the problems have the same structure. It is fully expected that students will discuss the WebWorK problems in groups, practice their skills by doing, and solidify their understanding by teaching one another. In this aspect, WebWorK is similar to conventional homework delivery methods. However, students cannot simply copy answers from their classmates. Thus, completing homework assignments in WeBWorK will lead students to become more effective problem solvers. While attempting problems, they are not able to look at the "answer in the back of the book" until they have discovered it through learning how to do the problem. This leads a student to feel more responsible for their own learning in a course because they are required to understand the material so they can answer their individual questions. An early WeBWorK study found that the immediate feedback feature was the most strongly endorsed benefit by students ${ }^{4}$. WeBWorK also supplies direct communication with the instructor via email from inside any WeBWorK homework problem, through an "Email the instructor" link contained in each problem, and allows access to discussion boards. A sample problem written for Circuits is shown in Figure 1.

One important note is how calculated answers with roundoff errors are entered into WeBWorK. Each problem has an error tolerance that is specified by the problem coder. Students should be instructed to maintain several decimal digits throughout their calculations to avoid excess roundoff errors. The default error tolerance for numerical comparisons is $0.1 \%$. However, the coder may also adjust the acceptable error tolerance for individual problems or for an entire course to be more forgiving or more stringent, depending upon the specific situation.

\section{Learning Impacts from Prior Studies}

There are numerous benefits to using WeBWorK over traditional paper-graded homework, from the perspective of student learning as well as regarding faculty and administrative resources. WeBWorK offers students real-time feedback on each problem by telling a student-user whether their response is correct or incorrect. Research has shown that prompt feedback enhances student learning. Students may also be given the opportunity of attempting a problem multiple times at the discretion of the instructor. One WeBWorK study found a correlation between student attempts and problems solved of 0.944 , "suggesting that once students began a problem they persisted until they had solved it" . Providing students the opportunity and encouragement to "continue working on a task until it is completed and accurate" also enhances student learning and achievement?

Studies on student performance and online/offline tutorial services indicate a correlation between online tutoring and student success. Data from one study conducted at Louisiana Tech in 2008 
In the circuit below, $i 1=12 \mathrm{~A}, \mathrm{i} 2=-10 \mathrm{~A}$, and $\mathrm{v} 1=6 \mathrm{~V}$.

Use nodal analysis to find $V_{x}$ in the circuit below
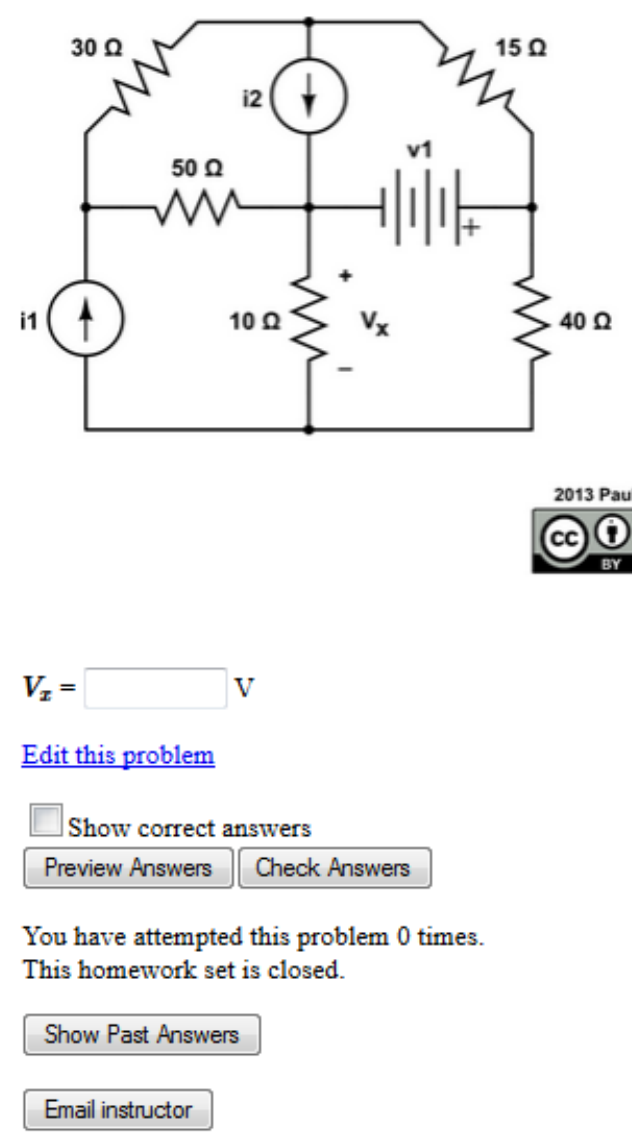

\section{Figure 1: Sample Homework Problem from Circuits Course}

revealed that students utilized online tutoring far more often than traditional tutoring programs. This information suggests a student preference to online technology ${ }^{8}$. A meta-analysis conducted by the Department of Education found that, "on average, students in online learning conditions performed modestly better than those receiving face-to-face instruction"9. Similar results were found in a study of college algebra students at a community college ${ }^{10}$. Specifically, online homework was found to be "just as effective as textbook homework in helping students learn college algebra and in improving students' mathematics self-efficacy," as measured by the Mathematics Self-Efficacy Scale. Further, it was observed that "online homework may be even more effective for helping the large population of college algebra students who enroll in the course with inadequate prerequisite math skills." Some universities report that students perform better on exams when using WeBWorK thus boosting student performance ${ }^{11}$. In most cases, the improvement was small, but nonetheless statistically significant compared to classes without WeBWorK ${ }^{6}$.

One study found that student preferences for online homework over traditional homework transcended gender, academic rank, and learning style, suggesting a diverse group of students 
may react positively to and benefit from online homework ${ }^{12}$. Another study related to middle school mathematics students found that "females expressed stronger opinions on the fact that instant scores and feedback helped them overcome difficulties in mathematics problem solving" "13. An NSF-funded global experiment, involving students and faculty from three continents, extended WeBWorK usage into Computer Science ${ }^{14}$. Student and faculty response was been positive, barring lack of consistent access to internet and computers, with the authors noting that "Systems such as WeBWorK offer the potential to transfer knowledge and teaching practices from one country to another."

\section{Current Study from Introductory Circuits}

The objective of the study was to investigate the effectiveness of homework done through WeBWorK on student development and learning, as opposed to traditional homework practices. Multiple studies have been done in the area of mathematics, but this experiment expanded it to the discipline of engineering. The course chosen for the study was an introductory circuits course-Electrical Engineering and Circuits I (ENGR 221). This course is a requirement for all engineering students at Louisiana Tech and covers such topics as the following: fundamental concepts (like current, voltage, and resistance), units and laws; network theorems and network simplification; phasors and AC solution of circuits; and power and electronic applications.

During the AY 13-14 Winter quarter, the two sections of the course offered were officially numbered as Sections 001 and 002 and will be referred to as such, henceforth. Section 001 was taught at 8:00 a.m., and Section 002 at 2:30 p.m. The sections were each taught by different professors; however, all the material — notes, tests, assignments, labs — were all identical. This ensured that each student was given equal information and evaluation across both sections.

To assess the student learning impact of WeBWorK, a control group was established through the following process. For a particular homework assignment that was a fairly isolated topic within the course, one section was required to do only paper homework, namely the instructor's printed WeBWorK assignment. The "paper only" homework section was not given access to that homework assignment in WeBWorK. The other section of the course completed homework on WeBWorK as usual. During the class period when the homework assignment was due, the same quiz was administered to both sections of the course.

The distribution of paper homework versus WeBWorK alternated between the two sections for each different topic. When one section offered paper homework, the other section offered the standard WeBWorK set. This process would switch for the next topic. Each student was given the same amount of time and resources to complete the homework. The primary difference between the paper homework and WeBWorK was the students' ability to receive immediate feedback on the correctness of a problem. On the virtue of paper homework, a student had little feedback to determine if they completed the proper steps to arrive at the correct solution. All other homework for the course was based in WeBWorK, with instructors also collecting a notebook at the end of the quarter containing all of the homework problems worked out in a typical engineering format. The homework notebook was graded on the formatting of problem statements and solutions but not on the correctness of the solution itself. 
For the AY 13-14 winter quarter, the quizzes covered three fundamental topics from introductory circuits - Nodal Analysis, Mesh Analysis, and Operational Amplifiers. That is, each topic was covered for an entire lecture, homework was given the same day, and then a quiz was given the following lecture.

Quiz Guidelines

The quiz was given at the start of the lecture. Before the quiz was given, however, homework was discussed and any questions surrounding the homework were answered. Students had the flexibility to ask anything regarding the homework, which included working sample problems. This session lasted approximately 7-10 minutes. After the completion of the question-and-answer session, the quiz was administered (closed book/closed notes). Each student was given approximately 10 minutes to complete analysis on a single circuit that covered the topic at hand. At the conclusion of this timeframe, the quizzes were collected and the solution was given.

Each of the quizzes were collected between the two sections and sorted based on the quiz topic. Among the participants in the course, only the students who had completed all three quizzes were calculated in the evaluation process. This comprised approximately 60 entrants in the review. Initially, an unbiased grader was designated to grade the quizzes; however, due to time constraints and lack of resources, each of the quizzes was graded by the professor of record according to a common rubric. The statistics from these quizzes were tabulated and analyzed.

Results

The two course sections started with 39 students each. Throughout the quarter, not all of the students were in attendance to take each of the quizzes. To make a more equitable comparison of quiz results, only students who were able to attend all 3 quizzes were used. This reduced the sample size to 31 and 25 for each of the two sections.

The average grade for each section on each quiz is shown in Table 1 below. The quizzes are highlighted to show which of the two sections received paper based or WeBWorK based homework. Section 001 is highlighted in red and Section 002 is highlighted in blue.

\begin{tabular}{|lcccc|}
\hline & Quiz 1 & Quiz 2 & Quiz 3 & Avg \\
\hline Paper & 0.842 & 0.736 & 0.645 & $\mathbf{0 . 7 4 1 2}$ \\
\hline WeBWorK & 0.732 & 0.863 & 0.630 & $\mathbf{0 . 7 4 1 6}$ \\
\hline Avg & $\mathbf{0 . 7 8 7}$ & $\mathbf{0 . 7 9 9}$ & $\mathbf{0 . 6 3 8}$ & $\mathbf{0 . 7 4 1 4}$ \\
\hline
\end{tabular}

Table 1: Quiz Score Averages by Section and Homework Type

The first item worth noting in Table 1 is the average scores for both WeBWorK and traditional paper homework are virtually identical. The WeBWorK scores were slightly higher, but not enough to be statistically significant. The data do support the conclusion that WeBWorK is equally effective as traditional paper based homework for student learning. 
The second item worth noting is the two sections had a significant performance difference. Section 001 had an average grade across all quizzes of 0.699 compared to 0.783 for Section 002 . This is likely due to the makeup of students in each section and the time difference between when the two sections met for class. The lower performing section was the earlier 8 AM class when more students were apt to miss class, show up late, and be less engaged during the class lectures. The importance of the difference between the two sections is reflected in the uneven distribution of homework assignments. The section that performed better was assessed twice with paper homework while the section that performed worse was assessed twice with WeBWorK homework. This would indicate the difference between paper-based homework and WeBWorK-based homework may be larger than directly indicated by the score averages we obtained. If only two quizzes are used for comparison to give an equal distribution between the two sections with paper and WeBWorK, the difference increases to 0.008 (using only quizzes 1 and 2) or 0.056 (using quizzes 2 and 3). With the small sample size of both sections, even these greater grade differences are not sufficient to show a statistically determinant improvement in learning comprehension with WeBWorK based homework. However, the case for showing WeBWorK is at least equivalent to paper based homework is more firmly established.

Assessing the learning performance of a homework system should not solely be based on the average grades received. The variance of grades should also be considered to determine how effective the learning system is at reaching all students. If a homework system was able to improve the scores of a select group of students while hindering the learning of another group of students of equal size, the averages grades would remain the same. This division would show up as an increase in the variance of the quiz grades. Table 2 below shows the variance of quiz scores for each section on each quiz. The quizzes are highlighted to show which of the two sections received paper based or WeBWorK based homework. Section 001 is highlighted in red and Section 002 is highlighted in blue.

\begin{tabular}{|llllc|}
\hline & Quiz 1 & Quiz 2 & Quiz 3 & Avg \\
\hline Paper & 0.0231 & 0.0899 & 0.1139 & $\mathbf{0 . 0 7 5 6}$ \\
\hline WeBWorK & 0.0612 & 0.0340 & 0.0333 & $\mathbf{0 . 0 4 2 9}$ \\
\hline Avg & $\mathbf{0 . 0 4 2 1}$ & $\mathbf{0 . 0 6 1 9}$ & $\mathbf{0 . 0 7 3 6}$ & $\mathbf{0 . 0 5 9 2}$ \\
\hline
\end{tabular}

Table 2: Quiz Score Variances by Section and Homework Type

The WeBWorK based homework had a smaller variance of grade scores, showing the class as a whole performed more cohesively. The variances, while overall seem to favor WeBWorK, do have some conflicting results. Over the first two quizzes they show a similar behavior as the score averages between the two sections, namely that Section 001 performed significantly worse than Section 002. The third quiz wildly diverges from this pattern. This outlier on Quiz 3 seems to overinflate the advantage of WeBWorK when looking at the average variances across all three quizzes. When considering only the first two quizzes, the variance between WeBWorK and paper homework is virtually identical, with the WeBWorK based homework having a slim advantage. Those results seem to coincide with the previous analysis. 
Looking at the variance of quiz scores, we can conclude as we did before, that WeBWorK is at least equivalent to paper homework for learning. This may be a more important result than the previous because it shows that for engineering students, the online based homework does not leave any portion of the class behind due to technological difficulties or comprehension. While this may not be a surprising result, it is reassuring verification that the current generation of engineering students is able to utilize and learn from online based educational tools.

\section{Student Reactions to WeBWorK}

Louisiana Tech has been using WeBWorK in mathematics courses for nearly nine years. Students have become quite accustomed to its usage in engineering courses as well. While new student users may complain about the input of answers into WeBWorK, when pressed for an opinion, they typically comment on the value of WeBWorK's features like the email instructor option on each problem, immediate problem feedback, and the ability to attempt a problem numerous times. Of course, these are only anecdotal comments. The current research study is in its early stages, and it will access student learning as well as student satisfaction.

\section{Conclusions and Future Directions}

In this work we have reported the results of a study to determine the impact of online homework when compared to traditional "paper" homework on student learning in an introductory circuits course. The data suggest that the online homework, administered through the open-source WeBWorK, is at least comparable to paper homework for student learning. This is consistent with what other studies involving online homework in mathematics have revealed. The authors acknowledge that some of the quiz scores in this study may have been skewed higher due to the professors going over homework questions prior to the quizzes. To strictly assess the impact of the differences in homework format, it would be more appropriate to administer the quizzes prior to answering any homework questions. This modification will be made in future quarters of the study. Additionally, four quizzes will be administered in future quarters so that all students are assessed the same number of times with quizzes post-"paper" homework and post-electronic homework. Finally, as the NSF-sponsored project includes developing online homework for courses in Statics and Mechanics of Materials and Thermodynamics, future educational studies to assess the impact of online homework on student learning in these courses will also occur.

\section{References}

[1] MAA-WeBWorK Web Page, [Online]. Available: http://webwork.maa.org/. [Accessed 24 January 2015].

[2] E. Brauer, "WeBWorK Development in Electric Circuits," in 2008 American Society for Engineering Education Pacific Southwest Annual Conference, 2008.

[3] H. Trussell, "Automating Analytical Homework," Proceedings of the IEEE, vol. 103, no. 1, pp. 2-7, January 2015.

[4] M. E. Gage, A. K. Pizer and V. Roth, "WeBWorK: Generating, Delivering, and Checking Math Homework via the Internet," in ICTM2 International Congress for Teaching of Mathematics at the Undergraduate Level, Hersonissos, Crete, Greece, 2002.

[5] R. L. Bangert-Drowns, C.-L. C. Kulik, J. A. Kulik and M. Morgan, "The instructional effect of feedback in test- 
like events," Review of Educational Research, vol. 61, pp. 213-238, 1991.

[6] L. Hirsch and C. Weibel, "Statistical Evidence that Web-Based Homework Helps," MAA Focus, p. 14, February 2003.

[7] R. J. Marzano, D. J. Pickering and J. E. Pollock, Classroom instruction that works: Research-based strategies for increasing student achievement, Alexandria, VA: Association for Supervision and Curriculum Development, 2001.

[8] J. P. Carpenter and B. D. Camp, "Using a Web-Based Homework System to Improve Accountability and Mastery in Calculus," in 2008 ASEE Annual Conference \& Exposition, Pittsburgh, 2008.

[9] B. Means, Y. Toyama, R. Murphy, M. Bakia, K. Jones and Center for Technology in Learning, "Evaluation of Evidence-Based Practices in Online Learning: A Meta-Analysis and Review of Online Learning Studies," U.S. Department of Education, 2010.

[10] D. S. Brewer, The Effects of Online Homework on Achievement and Self-efficacy of College Algebra Students, Utah State University, 2009.

[11] W. Ziemer, "WeBWorK: An Open-Source Online Homework System," in Invention and Impact: Building Excellence in Undergraduate Science, Technology, Engineering and Mathematics (STEM) Education, NSF DUE in collaboration with EHR and AAAS, 2004, pp. 169-171.

[12] D. Doorn, S. Janssen and M. O’Brien, "Student attitudes and approaches to online homework," International Journal for the Scholarship of Teaching and Learning, vol. 4, no. 1, January 2010.

[13] D. M. Nguyen, Y.-C. J. Hsieh and G. D. Allen, "The impact of web-based assessment and practice on students' mathematics learning attitudes," Journal of Computers in Mathematics and Science Teaching, vol. 25, no. 3, pp. 251-279, 2006.

[14] O. Gotel, C. Scharff, A. Wildenberg, M. Bousso, C. Bunthoeurn, P. Des, V. Kulkarni, S. Palakvangsa Na Ayudhya, C. Sarr and T. Sunetnanta, "Global Perceptions on the Use of WeBWorK as an Online Tutor for Computer Science," in 38th ASEE/IEEE Frontiers in Education Conference, Saratoga Springs, New York, 2008.

\section{Acknowledgements}

This material is supported by NSF DUE Grant \#1244833. Any opinions, findings, and conclusions or recommendations expressed are those of the authors and do not necessarily reflect the views of the National Science Foundation. 